\title{
HTLV-1 Infection
}

National Cancer Institute

\section{Source}

National Cancer Institute. HTLV-1 Infection. NCI Thesaurus. Code C39292.

An infection that is caused by HTLV-1. 